\title{
The Efficacy of a Video Self-Modeling Intervention on Peer Social Initiation Skills of Children with Autism Spectrum Disorders (ASD)
}

\author{
Lema Kabashi, Ph.D. \\ Assistant Professor - Special Education, University of Wisconsin-La Crosse, WI, USA
}

Louise A. Kaczmarek, Ph.D.

Associate Professor - Instruction and Learning, University of Pittsburgh

\begin{abstract}
Social initiation skills are considered among the most challenging skills to acquire by children with Autism Spectrum Disorder (ASD). Generalization of social initiations is another related challenging area. Research indicates that when provided with appropriate interventions, children with ASD may enhance social initiation skills and generalize them across settings, people, and materials. This study implemented a multiple probe single subject design across three children with ASD to examine whether a video self-modeling intervention is effective in establishing a mand repertoire and other types of peer social initiations. Generalization and maintenance effects were measured in addition to the social validity of the video self-modeling intervention. While video self-modeling led to substantial improvements in almost all of the target behaviors for one participant, additional strategies were implemented for two other participants. Discussion of findings, study limitations, and future recommendations are addressed as well.
\end{abstract}

Keywords: autism, children, video self-modeling, mands, social initiations.

\section{Introduction}

Social skills impairments are a defining feature of Autism Spectrum Disorders (ASD). Social skills, which are necessary for interacting and communicating with other people, develop naturally throughout infancy and the early childhood years in typically developing children. However, for children with ASD engaging in social interactions does not occur naturally (Trent, Kaiser, \& Wolery, 2005), affecting not only their relationships with adults, but also with peers (Nikopoulos \& Keenan, 2007). Although some children with ASD may improve their social behaviors to some degree after the age of 5 , deficits in playing cooperatively, establishing peer friendships, and recognizing peer feelings and responses remain deficient throughout life (Oke \& Schreibman, 1990). In short, children with autism are unlikely to experience the benefits of developing and maintaining social relationships with other people, especially their peers, without specific interventions designed to facilitate such skills (Maione \& Mirenda, 2006).

Reciprocal social interactions require individuals to both initiate interactions as well as respond to the social bids of others. Children with autism typically respond to social bids more frequently and easily than they initiate social interactions (Tsao \& Odom, 2006). Low levels of social initiation means that children with autism: a) have fewer opportunities to seek out social and verbal learning experiences, b) miss constructive information derived from the environment, $c$ ) fail to request assistance when necessary, d) have less opportunities to attain the responses that typically are derived from social initiations, and e) have limitations communicating beyond what others are willing to provide to them (Hume, Loftin, \& Lantz, 2009). Consequently, when social initiations are minimal, deficits in communication and other areas of development are more likely to happen (Oke \& Schreibman, 1990).

Interventions to improve the social initiations of children with ASD have included, among others, mand training (Taylor et al., 2005) and video self-modeling (Bellini, Akullian, \& Hopf, 2007; Boudreau \& Harvey, 2013). Both interventions have the potential to establish social initiation skills because they both increase independence and generalization, facilitate responsiveness from peers, increase the frequency and appropriateness of social initiations, and can be easily implemented by teachers (Zanolli, Draggett, \& Adams, 1996). 
1.1. Mand Training. Mands are considered a type of social initiation and are typically referred as "requests," with the exception that a mand is controlled by a motivating operation (MO). According to Michael (1993) an MO is "an environmental event, operation, or stimulus condition that affects an organism's behavior by altering a) the reinforcing effectiveness of other events and $b$ ) the frequency of occurrence of that part of the organism's repertoire relevant to those events as consequences" (p.192). In order to use an MO when teaching mands, specific attention should be paid to the reinforcing effectiveness of those events by capturing or manipulating them.

Two studies, Pellecchia and Hineline (2007) and Taylor et al. (2005), taught mands to children with autism while incorporating social initiations to adults and peers. While Taylor and her colleagues (2005) manipulated the MO in order to increase social initiations of three children with autism toward their peers with autism, Pellecchia and Hineline (2007) investigated acquisition of mands for preferred items as well as generalization of skills across parents, instructors, siblings, and peers of three preschoolers with autism. Findings of both studies showed acquisition of mands, but they both recommended teaching mands separately to adults and peers since they failed to generalize skills from adults to peers. In addition, Taylor et al. (2005) suggested that social interaction behaviors are incorporated into mand training or embedded into reinforcers in order to increase the appropriateness of mands as well as facilitate other types social interaction skills.

1.2. Video Self-Modeling. Video self-modeling (VSM) is one of the types of video-based intervention in which the target individual observes himself performing targeted behavior(s) in a video and then is expected to imitate the behavior(s), previously viewed, in a similar situation (Bellini et al., 2007). Video self-modeling allows individuals with ASD to learn through visual means. A visual format, allows them to review cues, decrease reliance on prompts (i.e., teachers, adults), and increase independence (Ganz, Earles-Vollrath, \& Cook, 2011). Motivation is another additional factor that plays a critical role in the efficacy of the video self-modeling intervention. Watching one's own successful behaviors has a positive impact on attention and motivation to attend to the modeled behaviors (Bellini \& Akullian, 2007). Researchers report that most of the participants enjoy watching themselves in the video (Boudreau \& Harvey, 2013). In order for video self-modeling to be successful with individuals with ASD, two factors -- self-recognition and attention span -- play a critical role. Selfrecognition consists of the target individual recognizing oneself on the video, whereas the latter is concerned with the target individual staying attentive throughout the video (Buggey \& Hoomes, 2011).

Although there is ample research using various types of video-based instruction (e.g., video modeling, point-of-view video modeling) to improve the social initiations of children with autism (Kabashi \& Kaczmarek, 2016), there are seven studies that used video self-modeling to improve social initiations of children with autism. The majority of these explored social initiation skills by measuring physical and vocal initiations to peers (Bellini et al., 2007; Boudreau \& Harvey, 2013; Buggey, 2012; Buggey et al., 2011; Kabashi \& Epstein, 2017). One study (Buggey, 2005), however, targeted unsolicited verbalizations to both peers and staff and another (Wert \& Neisworth, 2003) investigated spontaneous requesting to adults.

Overall, results of these studies supported video self-modeling as an effective intervention for improving social initiations of children with autism. However, several studies failed to improve social initiation skills of all participants (Buggey, 2012; Buggey et al., 2011; Wert \& Neisworth, 2003). Researchers noted that these participants did not attend to the video and participants in Buggey (2012) study were three years old. This supports previous research that video self-modeling is effective for individuals who are able to recognize themselves in the video, attend to the video, and enjoy watching themselves in the video. Nikopoulos and Keenan's study (2007) supports these findings given that they attempted to increase the social initiations of a child with autism using video self-modeling after he failed to respond to video modeling where the adult was a model. However, this participant wasn't interested in watching himself in the video and did not attend to the video, so he still didn't make any improvements. As for the age, there should be further studies investigating the impact of age in children.

In regard to generalization of the skills, participants of two studies generalized social initiation skills across peers and settings (Wert \& Neisworth, 2003; Kabashi \& Epstein, 2017). Given that participants in Wert and Neisworth's study (2003) watched a video at home and they displayed target skills at the school, generalization effects across settings in addition to across people were reported. Participants in Kabashi and Epstein's (2017) study generalized the skills across settings, peers, as well as items.

As seen in the aforementioned studies, both manding and video self-modeling interventions were successful to a degree. The mand training was successful in establishing mands, but did not generalize across persons or generalize other types of social initiations; video-self-modeling was successful in facilitating initiations to peers in some children, but studies lacked 
investigation of generalization. Video self-modeling has never been used to train mands, specifically independent manding to peers. Consequently, the current study was designed to investigate the efficacy of a video self-modeling intervention to establish manding and other types of social initiations to peers in young children with ASD. More specifically, the purpose of this study was two-fold: first, to investigate the efficacy of video self-modeling on the frequency of mands to peers and other types of peer social initiations (i.e., greeting, commenting) in children with ASD, and second, to investigate the efficacy of video self-modeling on facilitating the transfer of treatment gains across people, settings, and materials. Permission to conduct this study was issued by the Institutional Review Board.

\section{Methods}

\subsection{Target Population and Selection}

The participants in this study were three preschool-aged children with ASD. Participants were selected in accordance with the following five criteria: the child a) had a diagnosis of autism spectrum disorders, b) the child was between 3 and 5 years of age, $c$ ) the child was able to say at least three-word sentences or use an Augmentative and Alternative Communication (ACC) device, d) the child had low or nonexistent levels of social initiation skills with peers, and e) the child was not able to produce independent mands for preferred items to peers.

Prior to the current investigation, each participant had received a diagnosis of autism autism and was enrolled in a reversed integration program for children with autism. Existing assessments were utilized to determine the approximate level of the participants' language and social development. To determine entry levels of the participants' manding skills (i.e., manding for preferred items), the Mand Section of the Verbal Behavior Milestones Assessment and Placement Program (VB-MAPP; Sundberg, 2008) was administered. The VB-MAPP contains 16 measurements of language and language related skills, mand being one of these measures, which are presented in a developmental sequence within 3 levels (i.e., Level I -III). The participants' mand repertoire in Level 1 (0-18 months) was assessed through 5 milestones, consisting of the ability to emit mands with and without prompts, the number of different mands emitted, and the level of spontaneity and generalization, In Level 2 (18-30 months) the participants' mand repertoire for requesting missing items and actions was assessed through 5 milestones as well as the number of words used to mand, the level of spontaneity, and the number of novel mands. Finally, Level 3 (30-48 months) assessed the participants' ability to mand for information, stop/remove undesirable activities, give directions, instructions, or explanations, and mand for others to attend to his own intraverbal behavior through 5 milestones (Sundberg, 2008). The scoring procedure for each level consists of $0,1 / 2$, or 1 point for each milestone. Hence, the score of 5 indicates that the participant met each milestone in that level. The VB-MAPP results for the participants' will be interpreted based on their level and their points within that level.

Tuan. Tuan was a 4-years and 10-months old at the beginning of the study. Vietnamese and English were the languages used at home. On the VB-MAPP Mand Section, Tuan displayed all the skills at Level 1, except the generalization of mands across people, settings, and reinforcers. In Level II, he met only 2 milestones, scoring $2 \frac{1}{2}$ out of 5 points. In this level he demonstrated the ability to mand for 20 different missing items only when prompted "What do you need?" which is permitted in assessing this milestone. He was able to mand for others to emit 5 different actions and also 3 different mands containing 2 words (e.g., my turn). He did not score more then 1 and 2 mands respectively in Level III. No questions for acquiring information or any other questions were displayed at any point during the assessment or the study.

Sam. Sam was a 4-years and 11-month old at the beginning of the study. The VB-MAPP Mand Section results were $4 \frac{1}{2}$ at Level I and $2 \frac{1}{2}$ at Level II. He was able to mand without being prompted by the question "What do you want?" He did not meet the milestone related to generalization of mands across people, settings, and reinforcers, scoring only a $1 / 2$ point out of 1. In Level II the child did not spontaneously emit different mands or novel mands. No questions to acquire information were displayed by the child at any time during the assessment.

Jeremy. Jeremy, aged 4-years, 2-months at the beginning of the study, met almost all the milestones at Level 1, scoring 4 $1 / 2$ out of 5 points on the Mand Section of the VB-MAPP. Using the AAC device, the child was able to emit mands when asked "What do you want?" Generalization of mands represented one of the areas of difficulty for him in this level. In Level II Jeremy scored 3 out of 5 points. However, given that he used the communication device, he communicated in phrases, hence emitted mands that contained 2 or more words (e.g., blow bubbles). Emitting novel mands was not feasible because of the limited access to words on the AAC device. He was observed asking questions to mand for preferred items toward 
adults (i.e., Can I play with iPad?). However, this question was asked using one button only. He did not demonstrate the ability to use separate buttons in his AAC device to ask questions, to request from his peers, or to acquire information.

Peers. Initially, three different peers were selected and trained, each to work with a different participant. Peers were selected by the classroom teachers based on the children's social skills. One of the peers was unable to participate due to an extended absence from the program. Consequently, one peer served as the partner for first two participants. The peer who was unavailable to participate served as the peer in the generalization sessions.

2.3. Pre-experimental procedures. A preference assessment was conducted in the training setting using a paired stimulus procedure (Lavie \& Sturmey, 2002) in order to generate a list of preferred items to be used in the mand training. The list was selected based on the information from interviews with the children's teachers and parents. A self-recognition assessment, as described in Spiker and Ricks' (1984) study, was also conducted in the training setting. The child sat at the experimenter's lap and faced the mirror for approximately 10 seconds. Then he was turned $180^{\circ}$ facing away from the mirror and engaged in play with his preferred toy items that were placed on a table within his reach. While playing with the toys, the experimenter wiped red makeup, concealed in a tissue, onto the child's nose. The experimenter turned the child around to face the mirror again. If the child touched his nose within 15 seconds, then the child demonstrated selfrecognition. Results showed that all three participants had self-recognition abilities. Finally, the participants' abilities to attend to the video were assessed and all of them were able to attend to a video of themselves during free play for at least 1 minute.

\section{Settings and Materials}

The study took place in reversed integration preschool for children with autism. The study was conducted in the participants' classrooms and a training setting. The classroom consisted of a room divided into a partitioned instructional area, a play area, a library, and a table area. The training setting consisted of a partitioned 3" $\times 4$ " section of the teacher's room. The classroom contained typical preschool equipment and materials (i.e., tables, chairs, toys). The training setting contained a child size and a chair for watching the video and a second table and two child sized chairs for the activity session.

Other materials consisted of a video camcorder, a laptop computer for video viewing, and food and toy reinforcers for peers and the participants. Three highly preferred toy sets for the participants were used during each session throughout the study (see Table 1). In order for the motivating operations to be present, preferred toy items were restricted to use in the study. Prior to the start of the study, four video vignettes were created for each participant. The participants themselves and their assigned peers were used as the models in the video vignettes. If at any point during the study the participant lost interest in playing with the preferred toy item displayed in the video vignette, another highly preferred toy set was identified and the video vignettes were modified accordingly.

Table 1. Preferred Toy Items for Each Participant

\begin{tabular}{|c|c|c|c|}
\hline \multirow[b]{2}{*}{ Participant } & \multicolumn{3}{|c|}{ Order of toy sets used in the video vignette \& activity session } \\
\hline & First & Second & Third \\
\hline Tuan & Train set ( 3 items) & Ice Cream toy set ( 4 items) & Garage \& Cars ( 3 items) \\
\hline \multirow[t]{2}{*}{ Sam } & Train set (4 items) & Poker chips \& Box ( 3 items) & String \& Beads ( 3 items) \\
\hline & & & Ball Maze (3 items) \\
\hline \multirow[t]{2}{*}{ Jeremy } & Ramp \& Cars (2 items) & $\begin{array}{l}\text { Fridge Phonics \& Letters } \\
(4 \text { items) }\end{array}$ & Matching Middles (4 items) \\
\hline & Garage \& Cars ( 3 items) & & Matching Middles ( 3 items) \\
\hline
\end{tabular}

Note. Sam lost interest in the third selected toy item, which was replaced with a new preferred toy, Ball Maze; Jeremy lost interest in the first selected toy item, which was replaced with a new preferred Garage and cars. The number of items for the first and the third set of toys changed for Jeremy accordingly. 


\subsection{Research Design}

A multiple probe design across participants was utilized to demonstrate experimental control. The video self-modeling intervention (i.e., independent variable) involved four different video vignettes, referred to as Condition video $A, B, C$, and D. Condition video $A$ displayed the target child manding for preferred toy items from the peer. Condition video $B$ displayed the target child greeting the peer and manding for preferred toy items. Condition video $\mathrm{C}$ displayed the target child greeting the peer, commenting on the toy item, and manding for preferred toy items toward the peer. Finally, Condition video D displayed the target child greeting the peer, commenting on the toy item, manding for preferred toy items, and commenting on the activity.

Given that video self-modeling alone was not successful for acquiring all target behaviors with all participants, additional strategies such as prompting, cuing, reinforcement were utilized. While prompting consisted of the experimenter verbally prompting the participant (e.g., "(Name) say Hi (Name of peer)", cuing consisted of the peer saying at the end of the activity "This was fun!" Reinforcement consisted of the experimenter granting a preferred item, edible or toy, after the child displayed the target behavior. These strategies were used alone or as additional strategies to the video self-modeling or to one another.

After the child watched the video vignette, an activity session took place in which the child's behavior was assessed. The following dependent variables were targeted in this study: a) independent manding for preferred toy items, b) greeting, c) toy comments, and d) activity comments. Independent manding was defined as the child requesting a preferred toy item using a mand frame "I want (item)" directed to the peer. Greeting was defined as the child using vocalizations, or pressing a button on the AAC device, such as "Hi", "Hello", "Hi/Hello (name)" directed to the peer. Commenting on toys was defined as the child expressing an opinion about the preferred toy items (i.e., "This (item) is cool!") directed to the peer. Finally, Commenting on the activity consisted of the child expressing enjoyment to the peer regarding the activity (i.e., "This was fun!"). Such initiations had to be clearly directed to a peer and distinguished from an ongoing interaction by a change in items/activities or a discontinuation of the previous interaction for at least 5 seconds.

Criterion Performance. After the child reached the criterion performance for one video condition, the child was transferred to the next condition. The criterion performance for Condition video A was set at independently manding 8 out of 10 trials for three consecutive sessions. After the child reached the criterion performance for Condition video $A$, the child was transferred to the Condition video B. Performance criterion-level for Condition video $B, C$, and $D$ consisted of the child displaying the targeted social initiation behavior (i.e., greeting, commenting on toys and activity) once per session for three consecutive sessions (see Figure 1).

Figure 1. Criterion performance for all conditions.

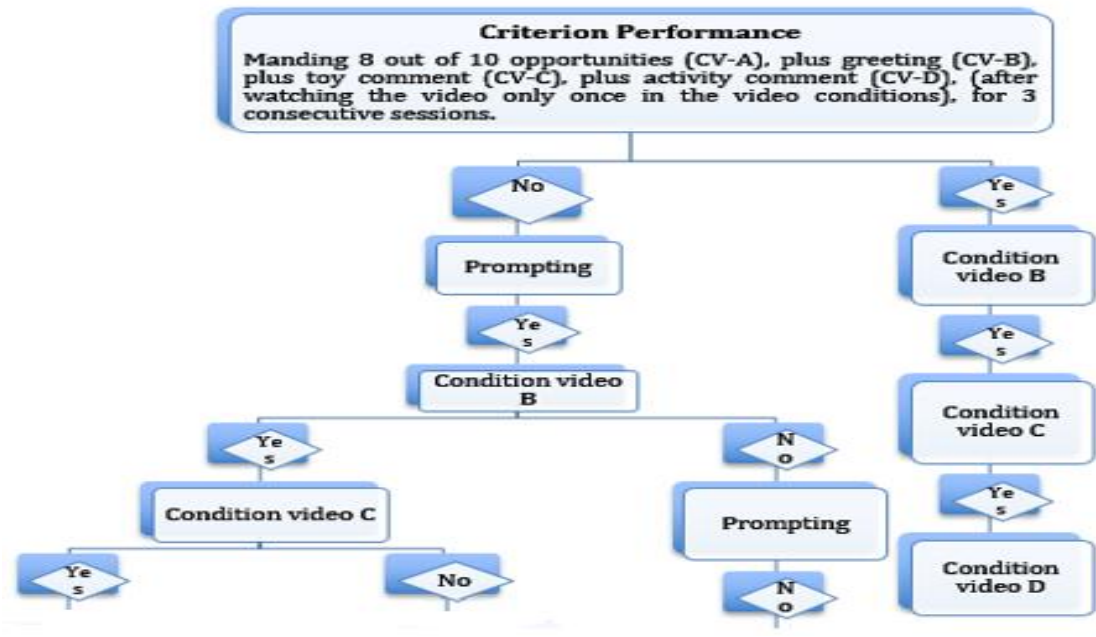




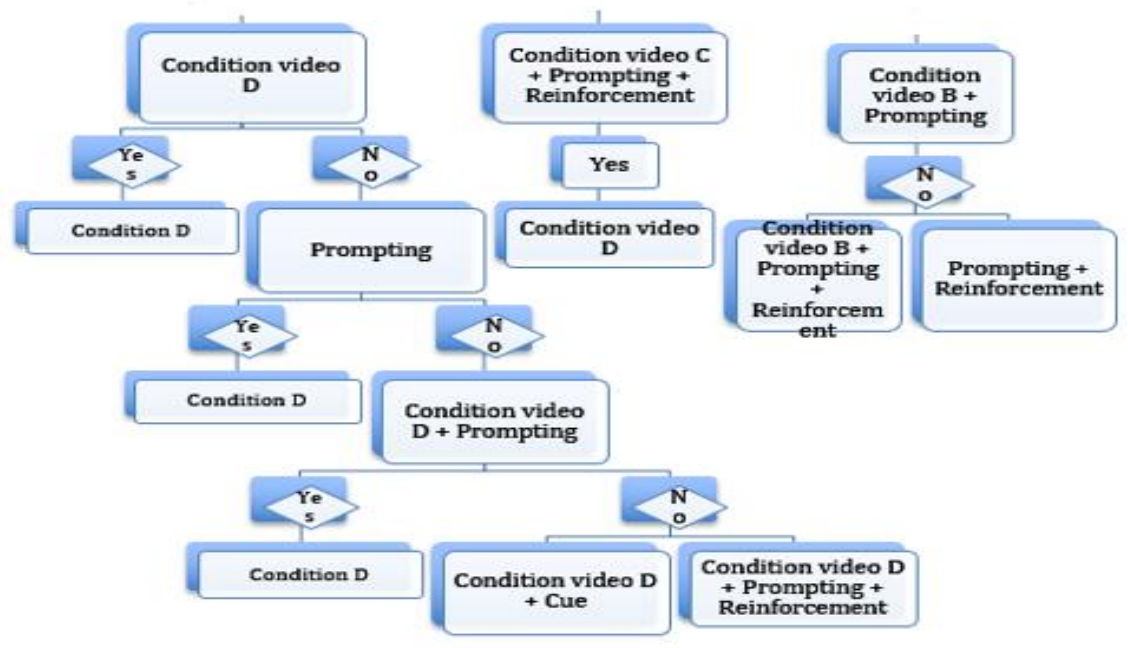

Procedures

Peer Training. Prior to the onset of the intervention, three typically developing peers were trained on verbal responses and play skills to use with the participants in the study. Peer-training session addressed a) the purpose of the intervention, $b$ ) watching video vignettes of the target behaviors displayed/not displayed, and c) practicing the targeted skills with the experimenter. Video modeling was utilized for training. A graduate student and the experimenter were used as models in the video vignettes. Peers watched the video and then role-played the scenario with the experimenter as displayed in the video vignette. The training continued in this fashion until the peers displayed $80 \%$ of the target behaviors for 2 consecutive sessions. Due to several of target behaviors that peers needed to display, if the target behaviors were displayed or not displayed by the participants, peer training continued for approximately two months.

Baseline. Baseline sessions were conducted for each participant in the absence of any video presentation. Participants' abilities to mand independently for preferred toy items as well as initiate other types of social behaviors toward peers were recorded. Baseline sessions were conducted both in the training and natural environments. In the training environment, the setting was arranged in the same way as it would be for the intervention activity sessions. The peer was seated at the table and while playing with the child's preferred toy items he commented "This (item) is cool! Three sets of preferred toy items consisting of a total of 10 toys/pieces of a toy set were placed on the table. The participant was provided with ten opportunities to mand. In a 10-min session, the number of mands, greetings, and comments displayed by each participant was tallied. In the natural environment, the baseline setting was arranged as it would be for the generalization sessions. This setting provided opportunities for the child to mand and engage in social behaviors with peers during a free play. Children were given the instruction "(Name) go play with your friends." The frequency of target behaviors in this 20-minute session was tallied.

Video self-modeling intervention. During the intervention, the children watched one video vignette every school day. At the beginning of the session the experimenter invited the child to watch a video (e.g., "(Name) let's watch the video"). Then, the child watched his own video vignette. The experimenter did not engage the child in conversation during the viewing of the video vignette other than redirecting if the child looked away from the video for more than 10 seconds by saying, "(Name), watch the video." After watching the video vignette, the experimenter said "(Name), let's do the same as in the video" and the activity session took place.

In the first video vignette, the peer was shown seated at a child size table. Three of the child's preferred toy items were placed on the table. As soon as the participant entered the room the peer who was playing with the toy item commented, "This (name of the item) is cool!" The participant approached the peer and sat on the chair already located near the table. The participant manded, "I want (item)." The peer said, "Sure" and gave him the toy item. The participant reached for the toy item and played with it. Then, the participant manded for the first piece of the toy set "I want (item)." The peer said, 
"Sure," and gave the participant the item. The child reached for the item and played with it or put the piece of the toy in the toy set. Finally, the child manded for the third item, "I want (item)." The peer said, "Sure" and gave the child the last piece of the toy set. The child reached for the item and played with it. Then, the peer said, "Let's play with something else." The participant then manded for the items of the second toy set. The same procedures were used for the third toy set.

The activity session for Condition video A was conducted in the same setting, using the same items, and with the same peer as displayed in the video, right after the participant watched the video clip. The experimenter invited the participant to the activity session by saying, "(Name), let's do the same as in the video." The activity scenario unfolded in the same manner as on the video vignette. The duration of the activity session depended upon the participant's performance. If the child manded 10 out of 10 opportunities toward the peer, despite the number of correct responses (i.e., displaying the mand frame "I want (item)"), then the activity session ended. If the participant did not mand for all toy sets then the duration of the activity session continued until the 10 minutes had elapsed. For the third participant 15 minutes time was allowed instead of 10 due to his use of an AAC device to communicate. The same scenario was applied for the other three condition videos, with the exception that in each activity session one more target behavior was added, accordingly to the video vignette displayed.

Condition D. This condition was conducted for three consecutive sessions to demonstrate the participants' ability to maintain the targeted behaviors without first watching the video. This condition was the same as Condition video $D$, except that the video was not shown Due to time limitations, Condition D was only conducted with the first and second participants.

Generalization condition. Five 20-minute generalization sessions for the first two participants and three for the third participant were conducted in the participants' classrooms during a free play activity. The participants' behaviors were observed in relation to the target behaviors during free play. In addition, five 10-minute generalization sessions mimicking the training setting were conducted in the participants' classrooms. However, the sessions involved untrained peers and novel toy items. If the child did not generalize the skills across all novelties (i.e., setting, peers, toys), then one of the components in the subsequent session reverted to that of the training setting (e.g., trained peer, different setting, different toy items). This process was repeated until generalization across novel peers, settings, and toy items was assessed.

Maintenance condition. The maintenance condition was conducted one month after the completion of the intervention. The three maintenance sessions, which were identical to baseline and generalization conditions, were conducted for three consecutive sessions for the first two participants and one session for Jeremy due to time constraints. The child was given the opportunity to mand for preferred toy items and to display other types of peer social initiations. There were no videos or cues provided at any time during the maintenance condition.

\subsection{Data Collection and Analysis}

Data Collection. All sessions were videotaped for subsequent data collection and reliability measurement. The experimenter was the primary coder who transcribed and scored occurrences of the dependent measures from all video recordings across conditions and participants. A graduate student served as the reliability observer and was trained using the adult video vignettes produced for peer training and those taken of peers during peer training. She was provided with a scoring manual containing operational definitions of the target behaviors, examples and non-examples of the target behaviors, and a scoring protocol. After an initial practice session during which she identified and recorded target behaviors while watching the aforementioned video recordings, she then recorded the target behaviors independently. Her recordings were compared to the experimenter's recordings until $90 \%$ agreement with the experimenter on three consecutive sessions was reached.

Interobserver agreement (IOA). The reliability observer reviewed randomly selected session videos, independently scoring $25-40 \%$ of sessions across each condition and each participant. The IOA was calculated by dividing the total number of agreements by the total number of agreements plus disagreements multiplied by 100 . The IOA for all participants was above $90 \%$, except for one session for Jeremy that was $89 \%$ (see Table 2). 
Table 2. Interobserver Reliability (IOA) and Procedural Fidelity (PF) and Reliability on Procedural Fidelity (IOA/PF) for Each Participant and Each Condition

\begin{tabular}{|c|c|c|c|c|c|c|c|c|c|c|c|c|c|}
\hline \multirow{2}{*}{\multicolumn{2}{|c|}{ Conditions }} & \multicolumn{4}{|c|}{ Tuan } & \multicolumn{4}{|c|}{ Sam } & \multicolumn{4}{|c|}{ Jeremy } \\
\hline & & Sessio & IOA & $\mathrm{PF}$ & $\overline{\mathrm{IO}} \mathrm{A}$ & Sessi & IOA & PF & $\overline{\mathrm{IO}} \mathrm{A}$ & & IOA & PF & $\overline{\mathrm{IOA}}$ \\
\hline \multirow{2}{*}{ 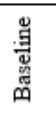 } & Manding & $25 \%$ & $100 \%$ & $100 \%$ & $100 \%$ & $29 \%$ & $100 \%$ & $100 \%$ & $100 \%$ & $33 \%$ & $100 \%$ & $100 \%$ & $100 \%$ \\
\hline & Free Play & $25 \%$ & $100 \%$ & $100 \%$ & $100 \%$ & $29 \%$ & $100 \%$ & $100 \%$ & $100 \%$ & $33 \%$ & $100 \%$ & $100 \%$ & $100 \%$ \\
\hline \multirow{2}{*}{ 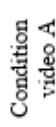 } & Condition video $\mathrm{A}$ & $33 \%$ & $100 \%$ & $100 \%$ & $100 \%$ & $30 \%$ & $100 \%$ & $100 \%$ & $100 \%$ & $33 \%$ & $96 \%$ & $100 \%$ & $100 \%$ \\
\hline & Prompting & $40 \%$ & $96 \%$ & $100 \%$ & $100 \%$ & N/A & & & & $33 \%$ & $100 \%$ & $91 \%$ & $98 \%$ \\
\hline \multirow{5}{*}{ 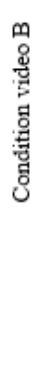 } & Condition video $B$ & $25 \%$ & $92 \%$ & $100 \%$ & $100 \%$ & $33 \%$ & $100 \%$ & $99 \%$ & $88 \%$ & $25 \%$ & $100 \%$ & $89 \%$ & $100 \%$ \\
\hline & Prompting & $33 \%$ & $100 \%$ & $100 \%$ & $100 \%$ & N/A & & & & $33 \%$ & $100 \%$ & $100 \%$ & $100 \%$ \\
\hline & $\begin{array}{l}\text { Condition video B + } \\
\text { Prompting }\end{array}$ & $25 \%$ & $96 \%$ & $100 \%$ & $100 \%$ & $\mathrm{~N} / \mathrm{A}$ & & & & $33 \%$ & $96 \%$ & $92 \%$ & $98 \%$ \\
\hline & $\begin{array}{l}\text { Prompting + } \\
\text { Reinforcement }\end{array}$ & $33 \%$ & $100 \%$ & $100 \%$ & $100 \%$ & N/A & & & & N/A & & & \\
\hline & $\begin{array}{l}\text { Condition video B + } \\
\text { Prompting + } \\
\text { Reinforcement }\end{array}$ & N/A & & & & N/A & & & & $33 \%$ & $100 \%$ & $92 \%$ & $96 \%$ \\
\hline \multirow{2}{*}{ 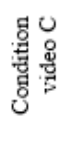 } & Condition video $\mathrm{C}$ & $40 \%$ & $94 \%$ & $100 \%$ & $94 \%$ & $25 \%$ & $100 \%$ & $100 \%$ & $100 \%$ & $33 \%$ & $100 \%$ & $90 \%$ & $98 \%$ \\
\hline & $\begin{array}{l}\text { Condition video C + } \\
\text { Prompting + } \\
\text { Reinforcement }\end{array}$ & $40 \%$ & $100 \%$ & $100 \%$ & $100 \%$ & N/A & & & & N/A & & & \\
\hline \multirow{4}{*}{ 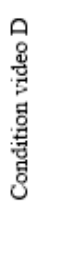 } & Condition video $\mathrm{D}$ & $33 \%$ & $97 \%$ & $100 \%$ & $99 \%$ & $40 \%$ & $100 \%$ & $100 \%$ & $100 \%$ & $33 \%$ & $100 \%$ & $95 \%$ & $100 \%$ \\
\hline & $\begin{array}{l}\text { Condition video } \mathrm{D}+ \\
\text { Prompting }\end{array}$ & N/A & & & & $33 \%$ & $100 \%$ & $100 \%$ & $100 \%$ & N/A & & & \\
\hline & $\begin{array}{l}\text { Condition video } \mathrm{D}+ \\
\text { Cue }\end{array}$ & $\mathrm{N} / \mathrm{A}$ & & & & $29 \%$ & $97 \%$ & $99 \%$ & $98 \%$ & $\mathrm{~N} / \mathrm{A}$ & & & \\
\hline & $\begin{array}{l}\text { Condition video D + } \\
\text { Prompting + } \\
\text { Reinforcement }\end{array}$ & $33 \%$ & $100 \%$ & $100 \%$ & $100 \%$ & $33 \%$ & $100 \%$ & $100 \%$ & $100 \%$ & $\mathrm{~N} / \mathrm{A}$ & & & \\
\hline \multicolumn{2}{|c|}{ Condition D } & $33 \%$ & $100 \%$ & $100 \%$ & $100 \%$ & $33 \%$ & $100 \%$ & $100 \%$ & $100 \%$ & $\mathrm{~N} / \mathrm{A}$ & & & \\
\hline \multicolumn{2}{|c|}{ Generalization } & $40 \%$ & $100 \%$ & $100 \%$ & $100 \%$ & $40 \%$ & $97 \%$ & $100 \%$ & $96 \%$ & $33 \%$ & $100 \%$ & $100 \%$ & $100 \%$ \\
\hline \multicolumn{2}{|c|}{ Maintenance } & $33 \%$ & $100 \%$ & $100 \%$ & $100 \%$ & $33 \%$ & $94 \%$ & $100 \%$ & $95 \%$ & $100 \%$ & $100 \%$ & $100 \%$ & $100 \%$ \\
\hline
\end{tabular}

Procedural fidelity. To determine whether the intervention procedures were being implemented accurately and consistently, procedural reliability data sheets were developed for all conditions. Procedural fidelity was calculated by adding total number of steps completed accurately divided by the total number of steps completed accurately plus the total number of steps completed inaccurately/missed multiplied by 100 . Reliability on procedural fidelity, using the same formula, was also conducted for $25-40 \%$ of sessions for each participant and each condition (see Table 2). 
Data Analysis. The effect of the independent variable on the dependent variables was determined through the visual inspection of the graphic representation of the data as well as by calculating the average level and analyzing the range level of independence for each dependent variable across each condition. The average level of independence for each dependent variable was calculated by summing the scores of the dependent variable for all sessions within that condition and dividing them by the total number of sessions conducted in that condition.

\section{Results}

The results of this study were analyzed by participant for each behavior targeted within each of the four video conditions (see Figure 2). The maximum number of mands was 10 mands per session. However, if the participant, for a variety of reasons manded independently to the peer more than 10 times those were also scored. Some of the reasons this happened included: the peer not granting the toy to the child within 5-sec of the participant's initial manding or the participant manding for a toy item that was reserved as a play item for the peer.

Figure 2. Number of independent mands, greetings, toy comments, and activity comments during the video self-modeling intervention alone and with other additional components

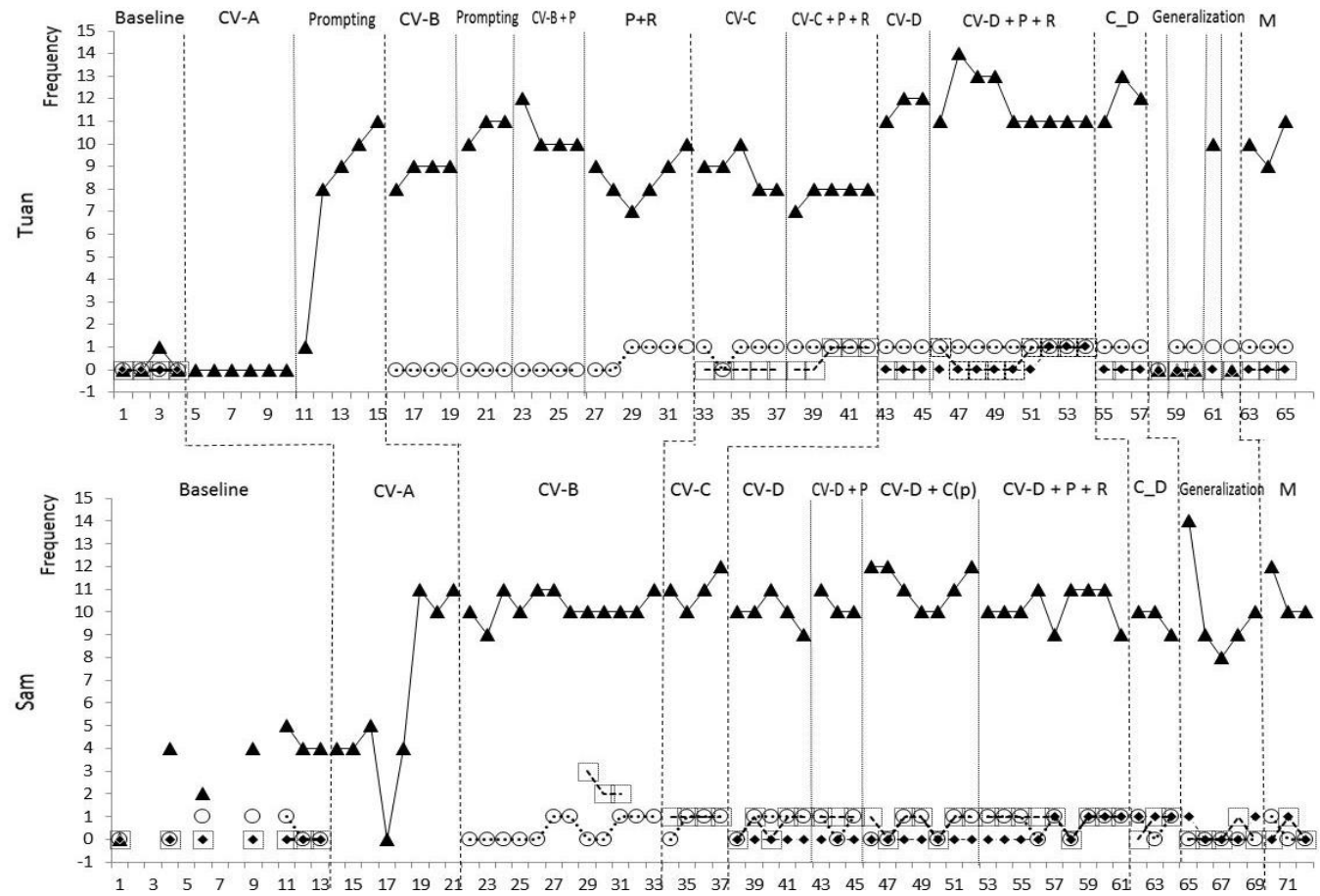




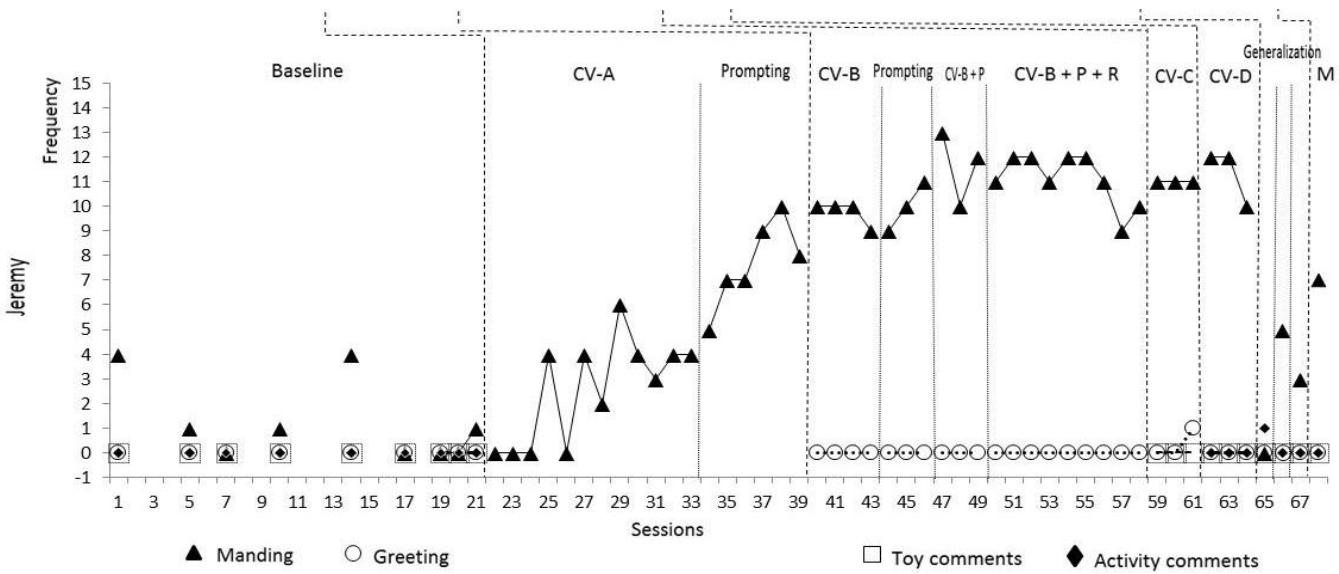

Note: $\mathrm{CV}-\mathrm{A}=$ Condition video $\mathrm{A}$; Prompting; $\mathrm{CV}-\mathrm{B}=$ Condition video $\mathrm{B}$; Prompting; $\mathrm{CV}-\mathrm{B}+\mathrm{P}=$ Condition video $\mathrm{B}+$ Prompting; $\mathrm{C} \_\mathrm{B}+\mathrm{P}+\mathrm{R}=$ Prompting + Reinforcement; $\mathrm{CV}-\mathrm{B}+\mathrm{P}+\mathrm{R}=$ Condition video $\mathrm{B}+$ Prompting + Reinforcement; $C V-C=$ Condition video $C ; C V-C+P+R=$ Condition video $C+$ Prompting + Reinforcement $C \mathrm{CV}-\mathrm{D}=$ Condition video $\mathrm{D}$; $C V-D+C=$ Condition video $D+$ Cue from the peer (on the video); $C V-D+P+R=$ Condition video $D+$ Prompting + Reinforcement; C_D = Condition D (no video), $M$ = Maintenance

Overall, the results demonstrated that all participants made substantial gains manding to peers for preferred toy items; however, the results for the three other target behaviors were not as consistent. All three participants displayed very few target behaviors during baseline sessions. Only Sam reached the criterion through video self-modeling alone. The two other participants required additional strategies (i.e., prompting, reinforcement, cuing), which were implemented alone or in combination with the video self-modeling, in acquiring the target behaviors. Table 3 portrays the average number of target behaviors for each participant by condition.

Table 3. Average Number of Target Behaviors for Each Participant by Condition

\begin{tabular}{|c|c|c|c|c|c|c|c|c|c|c|c|c|c|c|c|c|}
\hline \multirow{2}{*}{ Parficipants } & \multicolumn{4}{|c|}{ Mands } & \multicolumn{4}{|c|}{ Greetings } & \multicolumn{4}{|c|}{ Toy Comments } & \multicolumn{4}{|c|}{ Activity Comments } \\
\hline & $\mathrm{B}(\mathbb{m})$ & l(\#) & $G(\#)$ & $M(\#)$ & $\mathrm{B}(\mathbb{\#})$ & I(\#) & $G(\#)$ & $M(\#)$ & $\mathrm{B}(\mathbb{\#})$ & l(\#) & $\mathrm{G}($ (\#) & $M(\#)$ & $\mathrm{B}(\#)$ & I(\#) & $G(\#)$ & $M(\#)$ \\
\hline Tuan & $.25(4)$ & $8.66(53)$ & $2(5)$ & $10(3)$ & $0(4)$ & $0.67(42)$ & $0.6(5)$ & 1(3) & $0(4)$ & $0.32(25)$ & $0(5)$ & $0(3)$ & $0(4)$ & $0.25(12)$ & $0(5)$ & $0(3)$ \\
\hline Sam & $3.28(7)$ & $9.73(51)$ & $10(5)$ & $10.7(3)$ & $0.4(7)$ & $0.63(43)$ & $0(5)$ & $0.33(3)$ & $\alpha(7)$ & $1.03(31)$ & $0.4(5)$ & $0.33(3)$ & $0(7)$ & $0.26(27)$ & $0.4(5)$ & $0.33(3)$ \\
\hline
\end{tabular}

Note. $\mathrm{B}=$ Baseline, $\mathrm{I}=$ Intervention, $\mathrm{G}=$ Generalization, $\mathrm{M}=$ Maintenance \# = total number of sessions

Tuan. During baseline sessions in the training setting, Tuan manded once and displayed none of the other targeted behaviors. Within the 20-minute free play session, Tuan played alongside his peers but he did not display any of the target behaviors.

During the video self-modeling intervention sessions in Condition video A, Tuan did not display any independent mands to the peer for preferred toy items. After prompting without the video was implemented, he reached criteria for manding independently within four sessions.

After watching the Condition video B vignette for four sessions, Tuan did not display greeting behavior toward the peer. Subsequently, prompting only and video self-modeling plus prompting were tried for three and four sessions respectively, but without success. It was only after using prompting plus reinforcement without the video that Tuan finally greeted the 
peer independently when he entered the room and reached criterion. He was rewarded with edibles by the experimenter for the behavior displayed. His level of mands remained at or above criterion except for one session during each variation in procedure.

During the five Condition video $\mathrm{C}$ sessions (video only), Tuan did not make any independent comments on the toy item(s), but did continue to display independent mands and independently greeted the peer. Since prompting plus reinforcement had been successful in establishing the greeting, the video plus prompting and reinforcement were implemented next. In this variation, Tuan started commenting on the toy items and continued to maintain greeting and manding for preferred toys.

In the final condition, Condition video D (video only), Tuan did not start to comment on the activity nor did he maintain commenting on the toy. However, he continued to greet the peer and to mand at criterion. After prompting and reinforcement were added to the activity session following viewing the tape, Tuan reached criterion on the four target behaviors.

Results for Condition $\mathrm{D}$ to assess the participant's ability to display the targeted behaviors in the absence of the intervention procedures (i.e., video, prompting, reinforcement) showed that Tuan maintained independent mands and independently greeted the peer at criterion levels, although he did not display either type of comment.

Generalization was conducted for five days and included four variations. In the first variation, which consisted of three novelties (peer, setting, toys), Tuan did not display any of the target behaviors. In the next variation, which assessed two novelties (setting, toys) with the peer who participated in the training, Tuan greeted the peer, but did not display any of the other target behaviors in two consecutive sessions. In the third variation, which assessed two novelties (peer, setting) with the toys used in training, Tuan displayed 10 independent mands to a novel peer for preferred toy items but no other target behaviors. In the fourth variation, which assessed two novelties (peer, toys) in the training setting, Tuan only greeted the peer. Generalization was also assessed in the free play setting in conditions analogous to baseline. In five 20-minute sessions, Tuan displayed none of the targeted behaviors. Anecdotal notes from classroom teachers and other professionals indicated that after Tuan learned the greeting during training, he started to generalize greeting behavior to peers when coming to school in the morning.

One month after the completion of the intervention three maintenance sessions were conducted in the training setting with another three in the classroom. Results showed that Tuan maintained greeting and independent mands to the peer at criterion level for the sessions in the training setting. In the classroom, although greeting was maintained at the criterion, independent mands were not. No comments on toys or the activity were displayed in either setting.

Sam. During baseline, Sam independently manded to a peer with only one of the three sets of toys (range $0-5$ times per session) and greeted the peer a total of three times in the seven sessions. Commenting on the toy items and on the activity were not displayed. In the free play session, Sam did not initiate any of the target behaviors with his peers.

In the Condition video A sessions, Sam independently manded to the peer, but only for the same set of toys as in the baseline. When this favorite toy was removed (Session 4), his mands decreased to zero. In the next session, this highly preferred toy item was brought back and based on a multiple-stimulus preference assessment, one new toy set was substituted for one of the two less preferred original toy sets in this session and those remaining. After the substitution, he started to mand for items in all three toy sets, reaching criterion level.

In the Condition video B, Sam reached criterion on greeting during sessions $10-12$ and also maintained manding at criterion during the duration of the condition. Sam also commented on the toy items, 3 times in session 8 and 2 times each in sessions 9 and 10. Although Sam did not see himself on the video commenting on the toy items in this condition, the peer in the video always started the session by commenting on the toy item.

After viewing the Condition video $\mathrm{C}$ vignette for the first time. Sam commented on the toy item in the activity session and in each of the remaining sessions. He also maintained greetings to the peer in every session but the first and manded for preferred toy items at criterion.

After five sessions in Condition video D, Sam did not comment on the activity. Given that the target behavior was not acquired, video self-modeling plus prompting was implemented. However, even with the added prompting, Sam continued to score zero independent comments. Based on the assumption that Sam's spontaneous toy comments in Condition video 
B were imitations of the peer, the video was modified to include the peer saying "This was fun!" However, Sam still scored zero independent activity comments, so prompting and reinforcement were added to the activity session. In the fifth session of this variation, Sam finally started to display activity comments. The other three target behaviors were maintained throughout with manding at criterion, but with less consistency for greetings and toy comments.

In Condition D, Sam displayed all four target behaviors with manding and activity comments at criteria level for each of the three sessions.

Generalization of skills across three novelties (setting, peer, toys) was assessed for five sessions. Sam did generalize independent mands in this situation at criterion. He commented on the activity twice and on the toy item once, but did not greet his play partner. Results of five free play generalization sessions showed that Sam manded twice to the peer, but displayed none of the other target behaviors. Anecdotal notes from the classroom teachers and other professionals indicated that he generalized the greeting behavior across settings and individuals.

Three maintenance sessions in the training setting and three in the classroom were conducted one month after the intervention ended. Results revealed that Sam maintained manding at criterion in both settings. He maintained all three other targeted behaviors, although not consistently. The only greeting behavior that was displayed occurred in the first maintenance session in both the training setting and the classroom. Toy and activity comments were displayed in the training setting, but only activity comments were displayed in the classroom setting.

Jeremy. During baseline sessions in the training setting, Jeremy displayed a low level of independent mands to the peer (range $=0-4$ per session). No mands were displayed in the free play baseline sessions. No other target behaviors were displayed in the training or free play baseline settings.

In activity sessions following the viewing of the Condition video A vignette, Jeremy manded to the peer from zero to six times per session for the items in only one toy set except on one occasion when he displayed mands for two items in the second toy set. Since the objective was to mand for items within three different play sets, any additional manding for the same toy items above the required number were not scored. Given that he did not mand for other sets of available toys, prompting alone was implemented. During prompting alone, Jeremy reached the criterion for manding using all three sets of preferred toys.

Throughout four sessions of the Condition video B, Jeremy continued to mand to the peer for the toys at criterion, but never greeted the peer. Prompting without video, video with prompting, and video plus prompting and reinforcement were each implemented for at least 3 sessions. Although his mands to the peer remained at or above criterion, he never did display independent greeting. Due to time constraints, there were no additional components or other modifications made to establish greeting behavior.

Condition video $\mathrm{C}$ was conducted for three sessions only, also due to time limitations. Jeremy continued to mand above criterion, but never commented on the toy items. However, in the third session he greeted the peer independently.

Finally, in Condition video $D$, which was also conducted for three sessions only, Jeremy continued manding independently above criterion. However, none of the other targeted behaviors, including activity comments, were displayed.

Due to time constraints, Condition D was not conducted. The generalization condition, however, was conducted over three sessions. Given that there was no available novel trained peer, the generalization condition was conducted with the same peer that participated in the study. In the first session across two novelties (setting, toys), Jeremy did not display any mands, greeting behavior, or toy comments. However, he did comment once on the activity. In the second session, which assessed generalization across one novelty (setting), he displayed five independent mands, but no other target behaviors were displayed. In the final session, which assessed generalization in the training setting using novel toys, Jeremy displayed three independent mands. As for the generalization of target behaviors during the three free play generalization sessions Jeremy didn't display any of the target behaviors.

Due to time limitations and participant's absences, only one maintenance session was conducted. In this situation, Jeremy displayed seven mands but no other target behaviors. Four independent mands were the only behaviors displayed in the classroom. 


\subsection{Social Validity}

After the study was completed, 2 teachers, 2 assistant teachers, 2 related service personnel, and 3 parents of the children with ASD participated in assessing the social validity of the intervention. The professionals, who worked in the program, were asked to watch, two videos of each participant, one baseline and one intervention, and then complete a social validity questionnaire focused on the children's outcomes and the intervention characteristics using a 5-point Likert scale (1strongly disagree to 5-strongly agree). Parents watched two video vignettes of their own children, one baseline and one intervention, but completed the same questionnaire as the professionals. Video vignettes were randomly presented and there were no details provided of which video belonged to which condition (see Table 4 for results of both groups).

The professionals' responses on the questionnaire, although not everyone watched the videos of each participant, validated the success of the intervention. They agreed that the targeted behaviors were important skills, that the children enjoyed the sessions, and that they would recommend the intervention for other children. On the other hand, they were undecided as to whether the intervention was easily implementable. Professionals agreed that they had noticed the participants generalizing the target behaviors in different settings since their involvement in the study. They reported observing manding and greeting but no commenting on toys or activities.

Like the professionals, the parents validated the success of the intervention. They agreed more strongly than the professionals that the targeted behaviors were important skills, that the children enjoyed the sessions, that the intervention was easy to implement, and that they would recommend the intervention for other children. All three parents reported that they noticed the target behaviors at home and like the professionals, they reported observing manding and greeting but no comments on toys or activities.

\section{Discussion}

This investigation sought to determine the efficacy of video self-modeling in establishing peer social initiations in children with ASD. It was hypothesized that by watching themselves on video performing the target behaviors with peers, the participants would acquire those target behaviors. Video self-modeling led to substantial improvements in almost all of the target behaviors for one participant, Sam. The results of this study, however, might be considered inconclusive regarding the efficacy of video self-modeling alone to promote acquisition of manding and social initiation skills for the two other participants, Tuan and Jeremy.

Although video self-modeling alone was effective in establishing mands, greetings, and toy comments for Sam, intervention strategies such as prompting, reinforcement, and cuing were required in addition to the video self-modeling for the establishment of the fourth target behavior, activity comments. The results showed that prompting alone was effective in establishing a mand repertoire for both participants, Tuan and Jeremy, but not in establishing other target behaviors. Video self-modeling combined with prompting and reinforcement produced greater results in acquiring activity comments for Sam and toy comments and activity comments for Tuan. It is important to mention that video self-modeling alone or in combination with other strategies did not promote the acquisition of other target behaviors for Jeremy. The video selfmodeling intervention, however, is believed to have had some effect on the other intervention strategies used. For example, Tuan did not display any independent mands while in the video-self modeling intervention; however, after only one prompting session he displayed 8 out of 10 independent mands for preferred items to the peer.

The findings of the present study are noteworthy in that the target skills were maintained for two participants after the video self-modeling intervention was withdrawn. These results, especially Sam's, provide further support for the efficacy of video self-modeling for maintaining learned skills without the presence of the video. In addition, maintenance results for Tuan and Sam showed that they both maintained the target behaviors over time. Sam's maintenance of all target behaviors in both the training setting and the classroom supports previous findings (Nikopoulos \& Keenan, 2007; Wert \& Neisworth, 2003) that video self-modeling is an effective intervention for maintaining acquired skills over time. Tuan maintained manding for preferred toy items to the peer and greeting behavior in both settings; however, he did not maintain toy comments or activity comments. The maintenance results for Jeremy might be considered inconclusive given that his only maintenance session immediately followed generalization sessions.

In addition, the efficacy of video self-modeling for the generalization of skills across peers, settings, and materials is best supported by the performance of Sam. He was the only participant that transferred all the target skills, except greeting, to a novel peer, using novel toy items in a novel setting. Two other participants transferred manding skills into a novel setting 
when using the same toy items only. While in a training setting, manding skills were transferred to novel toy items for Jeremy, and greeting a novel peer for Tuan. Tuan also transferred greeting behavior to a novel setting but only with the peer who participated in the training. Jeremy's activity comment displayed in the novel setting and with novel toys should be interpreted with caution because he pressed a button to display activity comments only once during the intervention and that was after the peer commented on the activity first.

Indeed, the social validation data provided further evidence that the intervention was effective to improve social initiation skills of children with ASD. Both professionals and parents indicated that all three children demonstrated more appropriate greeting and manding skills during the intervention than in the baseline. Both respondents agreed that participants enjoyed video self-modeling sessions and recommended the intervention for other children with ASD. In addition, they indicated that the occurrence of manding and greeting behaviors was observed in classroom and home settings as a result of the intervention.

Overall, video self-modeling produced better outcomes for Sam compared to the other two participants. One speculation for such results is related to the child's characteristics. It was observed that Sam displayed characteristics of echolalia. He was the only child that also imitated the peer's behaviors seen in the video in addition to behaviors targeted for him. Other researchers have also pointed out that echolalia might be a possible explanation for the efficacy of video instruction on promoting acquisition of social skills (Charlop \& Milstein, 1989).

\subsection{Supporting the Use of Video Self-Modeling in Teaching Mand Repertoire}

For all participants, manding independently for preferred toys to the peer was acquired, generalized, and maintained more than the other target behaviors. There are a few explanations for these results. First, it is well established that the mand is distinguished from other types of language because it is controlled by motivational variables (Sundberg, 2004). The literature emphasizes deprivation as one of the most common motivating operations in establishing mands (Harman \& Klatt, 2005). It was suggested that an increase in the level of deprivation could possibly evoke specific behaviors (Sundberg, 2004). Additionally, teaching a child to mand with highly preferred items is more effective than using less preferred toy items (Harman \& Klatt, 2005). Given that the toy items used in this study were empirically-determined as highly preferred toys, it was more likely that deprivation would evoke their mands. Consequently, all participants did not have access to their preferred toy items at any time other than during the study session. During the study two of the participants changed their preferences for toy items. Sam lost interest in the third toy set (i.e., string \& beads) whereas Jeremy lost interest playing with the first toy set (i.e., ramp \& cars). Until the optimal sets of toys were determined, the frequency of mands remained low. It is worth mentioning that initially Sam was interested in playing with only one of his highly preferred toy items. When that toy was removed his mands to the peer decreased to zero. When the preferred toy item was returned and the nonpreferred toy item was replaced with a new preferred toy item he reached the criterion for that condition within 5 sessions. Acquisition of other target behaviors was not controlled by the motivating operation as was the case with mands. On the contrary, the participants were not as motivated by their peer's responses to display greeting or any of the commenting behaviors.

Second, the participants had more opportunities to observe themselves manding on the video and more opportunities to actually mand in the activity sessions than they had for the other target behaviors. The video showed participants having 10 opportunities to mand for preferred toys across three different toy sets. In the activity session, which followed immediately, the children were provided with 10 opportunities to mand for the same toy items in the activity session. This was not the case with other target behaviors. Based on the social appropriateness of the behaviors targeted in this study, greeting was displayed at the beginning, commenting on toys when starting to play with toys, and the last comment was displayed at the end of the activity. Consequently, three of these target behaviors were only observed once on the video and displayed only once in the activity sessions.

Third, the order of preferred toy items seemed to have an effect on manding. For two participants, Tuan and Jeremy, the second toy set was the most highly preferred toy item (i.e., ice cream, fridge phonics). The most preferred toy item for Sam was the first toy set (i.e., a train set). Tuan and Jeremy seemed to mand for the first toy set in order to play with their second toy set. While Tuan did not put away the ice cream when playing with the third toy set, Jeremy displayed a lower rate of independent mands for the third toy set compared to the first two toy sets. 
4.2. Implications for practice. The research findings of the present investigation have valuable implications for all parties involved in educating children with ASD. Although basic technology knowledge is needed to capture the video and edit the video vignette, innovation in video technology has greatly simplified the procedures for successfully designing and implementing the video self-modeling intervention. Professionals and family members may find this intervention attractive because through editing, they expose their children to verbal or motor behaviors that go beyond what they are demonstrating at the present time (Buggey \& Hoomes, 2011). However, constructing a video of target behaviors should be done with caution; new behaviors must be appropriate to children's development and ability levels.

As noted in the results of this study, all the participants were highly motivated to watch their own video vignettes. Accordingly, professionals who employ video self-modeling in their classrooms are more likely to find that children with ASD who meet two basic prerequisites -- self recognition and attention to the video (Bellini \& Akullian, 2007) -- will most likely be motivated to participate in the intervention as did all the participants in this study; hence transitioning challenges due to changes from one activity to another are less likely to occur.

4.3. Limitations. Given that the purpose of this study was to examine the efficacy of video self-modeling alone, additional intervention strategies (e.g., prompting, reinforcement, cuing) implemented in order to establish the target behaviors or reach the criterion performance may be considered as one of the study limitations. Although it is clear that the video selfmodeling was not successful in improving all the target behaviors for all the participants, the use of other intervention strategies makes it difficult to extrapolate exactly which part of the intervention produced more significant results.

Another potential limitation was the large number of sessions conducted in this study. This limitation might be considered in light of the fact that although the number of sessions ranged from 66 to 72 sessions for the participants, the duration of each session was 10 minutes or shorter for some conditions based on the child's performance within a session. In addition, the intervention itself was enjoyable for all of them and not once did they refuse or display non-compliant behaviors before or during the sessions.

Given that Jeremy used an AAC device as his communication mode whereas two other participants were verbal represented another limitation to the study. Although for the two other participants the video vignettes and the activity sessions were shorter, the lengths of the video vignettes for Jeremy were longer because they depicted him pressing the button and displaying the target behaviors. For the same reason, the activity sessions were also longer (i.e., 15-min).

The results of this study are limited in that the same conditions and the same number of sessions across conditions were not conducted with all the participants due to time constraints. For Jeremy, three sessions of Condition video $C$ and $D$ were conducted only whereas Condition $\mathrm{D}$ was not conducted at all. There were also only three generalization sessions and one maintenance session instead of five and three respectively, conducted with the other two participants.

Another possible limitation involves the time, approximately two months, required for peer training. Video modeling was utilized to train peers on the verbal and motor behaviors that they needed to display during the activity session if the target behaviors were displayed or not displayed by the child with ASD. Additional strategies (i.e., prompting, visual cues) during the training session might have reduced the time spent on peer training.

Creation of the video vignettes before the baseline condition might be considered a limitation. During the creation of the video vignettes for each participant prompting and reinforcement was involved for several days, approximately 10 minutes a day, in order for the participants to display the target behaviors clear enough so they would learn from watching their performance on the video vignette. The child might have picked up the target behaviors during the creation of the video and have displayed any of them during the baseline.

4.4. Future recommendations. Although there were many findings in this investigation, there are several issues that remain unanswered and in need of further investigation. First, there is a considerable body of literature that reported failure of video self-modeling or other types of video instruction for improving the social initiations of children with ASD. According to Bellini and Akullian (2007) attention and motivation are two essential features that contribute to the success of video selfmodeling. It was assumed that if the child does not attend to the video, it is less likely that he or she will imitate the behavior. Although all participants in this study were very attentive to the video and were highly motivated, the video self-modeling alone was not successful in promoting acquisition of the social initiations targeted in this study for all participants even though all had already shown the ability to recognize themselves. As a result, further research should be done on examining 
the characteristics and traits of children with ASD in order to determine who might benefit the most from the video selfmodeling intervention.

It is well established that video self-modeling is a promising intervention for children with ASD. This study, as other studies (Apple, Billingsley, \& Schwartz, 2005; Maione \& Mirenda, 2006), also utilized other intervention strategies in addition to the video self-modeling intervention. Given that the purpose of this investigation was not to compare the efficacy of video selfmodeling alone to video self-modeling with other additional intervention strategies, no conclusions can be made with certainty about the efficacy of video self-modeling alone or in combination with other strategies. Therefore, there is a need for comparative studies to be conducted in order to determine the efficacy of video self-modeling compared to video selfmodeling plus other additional components.

Further research is needed to investigate the efficacy of the video self-modeling intervention in the acquisition of mands for information. Research shows that the information itself is not motivating for children with ASD (Endicott \& Higbee, 2007). This intervention might be beneficial given that watching the video is motivating for most children with ASD.

Finally, it would be useful for research to assess the use of the video self-modeling intervention in establishing a mand repertoire in a natural environment instead of a structured environment. It would be of interest to investigate the efficacy of video self-modeling utilizing the newest technology (e.g., iPad, iPod) in a natural environment.

Findings of this study expanded the literature on video self-modeling in several ways. First, video self-modeling may be utilized to establish a mand repertoire with young children with ASD. Second, children with ASD may benefit from this intervention in regard to greeting their peers as well as commenting while playing with the peer. Third, children with ASD who display characteristics of echolalia seem to respond better to the video self-modeling intervention. Fourth, the video self-modeling combined with prompting and reinforcement might produce greater outcomes for children with ASD. Fifth, children with ASD who use an AAC device to communicate may also benefit from this intervention.

In summary, the most recent data on the prevalence of autism has been alarming, pointing out a real need for evidencebased interventions for promoting social initiation skills for children with ASD. Ready access and ease of use of today's technology provides greater opportunities for the video self-modeling intervention to be implemented with young children with ASD.

\section{References}

[1] Apple, A. L., Billingsley, F., \& Scharwtz, I. S. (2005). Effects of video modeling alone and with selfmanagement on compliment-giving behaviors of children with high-functioning ASD. Journal of Positive Behavior Interventions, 7(1), 33-46.

[2] Bellini, S., \& Akullian, J. (2007). A meta-analysis of video modeling and video self-modeling interventions for children and adolescents with autism spectrum disorders. Exceptional Children, 73(3), 264-287.

[3] Bellini, S., Akullian, J., \& Hopf, A. (2007). Increasing social engagement in young children with autism spectrum disorders using video self-modeling. School Psychology Review, 36(1), 80-90.

[4] Boudrey, J., \& Harvey, M. T. (2013). Increasing recreating initiations for children who have ASD using video self modeling. Education and Treatment of Children, 36(1), 49-60.

[5] Buggey, T. (2005). Video self-modeling applications with students with autism spectrum disorder in a small private school setting. Focus on Autism and other Developmental Disabilities, 20(1), 52-63.

[6] Buggey, T. (2012). Effectiveness of video self-modeling to promote social initiations by 3-year-olds with autism spectrum disorders. Focus on Autism and Other Developmental Disabilities, 27(2), 102-110.

[7] Buggey, T., \& Hoomes, G. (2011). Using video self-modeling with preschoolers with autism spectrum disorder: Seeing can be believing. Young Exceptional Children, (14)3, 2-12.

[8] Buggey, T., Hoomes, G., Sherberger, M.E., \& Williams, S. (2011). Facilitating social initiations of preschoolers with autism spectrum disorders using video self-modeling. Focus on Autism and Other Developmental Disabilities, 26(1), 25-36.

[9] Charlop, M. H., \& Milstein, J. P. (1989). Teaching autistic children conversation speech using video modeling. Journal of Applied Behavior Analysis, 22(3), 275-285.

[10] Endicott, K., \& Higbee, T. S. (2007). Contriving motivating operations to evoke mands for information in preschoolers with autism. Research in Autism Spectrum Disorders, 1, 210-217. 
[11] Ganz, J. B, Earles-Wollrath, T. L, \& Cook, K. E. (2011). Video modeling: A visually based intervention for children with autism spectrum disorder. Teaching Exceptional Children, 43(6), 8-19.

[12] Hartman, E.C., \& Klatt, K. P. (2005). The effects of deprivation, presession exposure, and preferences on teaching manding to children with autism. The Analysis of Verbal Behavior, 21, 135-144.

[13] Hume, K., Loftin, R., \& Lantz, J. (2009). Increasing independence in autism spectrum disorders: A review of three focused interventions. Journal of Autism and Developmental Disorders, 39, 1329-1338.

[14] Kabashi, L., \& Epstein, A. (2017). Improving social initiations of children with autism using video self-modeling with video feedback: A case study. Journal or Educational and Social Research, 7(2), 111-122.

[15] Kabashi, L., \& Kaczmarek, L. A. (2016). Evaluating the efficacy of video-based instruction (vbi) on improving social initiation skills of children with autism spectrum disorder (asd): A review of literature. Review Journal of Autism and Developmental Disorders. doi: 10.1007/s40489-016-0098-5.

[16] Lavie, T., \& Sturmey, P. (2002). Training staff to conduct a paired-stimulus preference assessment. Journal of Applied Behavior Analysis, 35(2), 209-211.

[17] Maione, L., \& Mirenda, P. (2006). Effects of video modeling and video feedback on peer-directed social language skills of a child with autism. Journal of Positive Behavior Interventions, 8(2), 106-118.

[18] Michael, J. (1993). Establishing operations. The Behavior Analyst, 16(2), 191-206.

[19] Nikopoulos, C. K., \& Keenan, M. (2007). Using video modeling to teach complex social sequences to children with autism. Journal of Autism Developmental Disorders. 37, 678-693.

[20] Oke, N. J., \& Schreibman, L. (1990). Training social initiations to high-functioning Autistic child: Assessment of collateral behavior change and generalization in a case study. Journal of Autism and Developmental Disorders, 20, 479-497.

[21] Pellecchia, M., \& Hineline, P. N. (2007). Generalization of mands in children with autism from adults to peers. The Behavior Analyst Today, 8(4), 483-491.

[22] Spiker, D., \& Ricks, M. (1984). Visual self-recognition in autistic children: Developmental relationships. Society for Research in Child Development, 55(1), 214-225.

[23] Sundberg, M.L. (2008). Verbal behavior milestones assessment and placement program: The VB-MAPP. Concord, CA: AVB Press.

[24] Sundberg, M. L. (2004). A behavioral analysis of motivation and its relation to mand training. In

[25] W.L. Williams (Eds.), Developmental disabilities: Etiology, assessment, intervention, and integration (pp. 199220). Reno, NV: Context Press.

[26] Taylor, B. A., Hoch, H., Potter, B., Rodriguez, A., Spinnato, D., \& Kalaigian, M. (2005). Manipulating establishing operations to promote initiations toward peers in children with autism. Research in Developmental Disabilities, 26, 385-395.

[27] Trent, J. A., Kaiser, A. P., \& Wolery, M. (2005). The use of responsive interaction strategies by siblings. Topics in Early Childhood Special Education, 25(2), 107-118.

[28] Tsao, L. L., \& Odom, S. L. (2006). Sibling-mediated social interaction intervention for young children with autism. Topics in Early Childhood Special Education, 26(2), 106-123.

[29] Wert, B.Y., \& Neisworth, J. T. (2003). Effects of video self-modeling on spontaneous requesting in children with Autism. Journal of Positive Behavior Interventions, 5(1), 30-34.

[30] Zanolli, K., Draggett, J., \& Adams, T. (1996). Teaching preschool age autistic children to make spontaneous initiations to peers using priming. Journal of Autism and Developmental Disorders, 26(4), 407-421. 